\title{
Editorial: Shifting from Technology-Enhanced Learning to Technology-Transformed Learning - Best Papers Selected from the Conference APTEL 2010
}

\author{
Gwo-Jen Hwang* \\ Chair Professor, Graduate Institute of Digital Learning and Education \\ National Taiwan University of Science and Technology, Taiwan \\ E-mail: gjhwang.academic@gmail.com
}

\section{Nian-Shing Chen}

Department of Information Management National Sun Yat-Sen University, Taiwan

E-mail: nschen@mis.nsysu.edu.tw

\section{Ju-Ling Shih}

Department of Information and Learning Technology National University of Tainan, Taiwan

E-mail: juling@mail.nutn.edu.tw

*Corresponding author

\begin{abstract}
In the past decade, the rapid development of computer and communication technologies brings many opportunities for developing innovative learning environments with rich resources. Technology enhanced learning shifted their focus from technology to support factual learning, memorization and the reinforcement of basic skills to stimulate students to engage in meaningful learning and situated learning. With the support of computer and communication technologies, students are able to develop higherorder skills, such as critical thinking and problem-solving skills individually or collaboratively. Technology enhanced learning has become an interdisciplinary issue that attracts researchers from various fields to work together.
\end{abstract}

Keywords: Technology Enhanced Learning; Technology Transformed Learning; Asia-Pacific

Biographical notes: Dr. Gwo-Jen Hwang is currently Chair Professor of National Taiwan University of Science and Technology Distinguished. He received his Ph.D. degree in Computer Science and Information Engineering from National Chiao Tung University in Taiwan in 1991. Dr. Hwang has plenty of academic research and administration experiences. He had been Director of Computer Center of National Chi Nan University for three years, Chairman of Computer and Education Department of National University of Tainan for one year, and Dean of College of Science and Engineering of National University of Tainan for five and a half years. Dr. Hwang has published more than 350 academic papers, including 120 papers in such professional journals as Computers \& Education, Educational Technology \& Society, British Journal of Educational Technology, Innovations in Education and Teaching International, 
Electronic Library, and IEEE Transactions on Education among others. One of his research papers has been recognized by the well-established Computers \& Education as one of the top 10 most frequently cited papers. Owing to the good reputation in academic research and innovative inventions of e-learning, in 2007 and 2010, he received the annual Most Outstanding Researcher Award from the National Science Council in Taiwan.

Dr. Nian-Shing Chen is a Distinguished Professor at the Department of Information Management in the National Sun Yat-Sen University, Taiwan. He has published over 330 papers in the international referred journals, conferences and book chapters. He is the author of three books with one textbook entitled "e-Learning Theory \& Practice". Prof. Chen received the distinguished research award from the National Science Council, Taiwan in 2008. His current research interests include assessing e-Learning course performance; online synchronous teaching \& learning; mobile \& ubiquitous learning; Natural user interface \& Game-based learning. Professor Chen is a member of IEEE, ACM and the Chair for Expert Seminars and Web-based Tutorials, the IEEE Technical Committee on Learning Technology (http://ttf.ieee.org/). He is a Co-Editors-In-Chief of the SSCI indexed Journal of Educational Technology \& Society.

Dr. Ju-Ling Shih is currently an Professor of the Department of Information and Learning Technology in National University of Tainan, Taiwan. She earned her Ed.D. in Communication and Education from Teachers College, Columbia University, specializing in distance education and instructional technology. Her research interests include instructional design and qualitative research in digital learning, digital games, mobile learning, and technologymediated education in various levels and fields. Dr. Shih received the $2012 \mathrm{Ta}-$ You Wu Memorial Award from National Science Council in Taiwan.

\section{Technology Enhanced Learning}

In the past decade, the rapid development of computer and communication technologies brings many opportunities for developing innovative learning environments with rich resources. Technology enhanced learning shifted their focus from technology to support factual learning, memorization and the reinforcement of basic skills to stimulate students to engage in meaningful learning and situated learning. With the support of computer and communication technologies, students are able to develop higher-order skills, such as critical thinking and problem-solving skills individually or collaboratively. Technology enhanced learning has become an interdisciplinary issue that attracts researchers from various fields to work together.

\section{Introduction to This Special Issue}

Technology enhanced learning has been recognized as being an important research topic in the fields of education and computer science. In the past decade, many studies concerning this topic have been reported by researchers all over the world. The Technology Enhanced Learning (TELearn) was held in the past several years since 2007. The purpose is to provide a public forum to gather international researchers who study 
technology enhanced learning for publishing research findings and exchanging experiences in their areas of interests.

First TELearn conference took place in Taiwan in 2007. Next year in 2008, TELearn conference was held in Vietnam. In the following year, 2009, the conference was held in Taiwan again. The majority of participants attending the series of TELearn conferences came from Asian-Pacific regions; therefore, the Organizing Committee of TELearn decided to change the title of the conference from Technology Enhanced Learning conference to Asia-Pacific Conference on Technology Enhanced Learning (APTEL) in 2010. APTEL 2010 was held in Kansai University in Japan. Its main theme of the conference was "Social Computing in e-Learning" with the following topics:

- Affective and Pervasive Computing for Learning

- Classroom, Ubiquitous and Mobile Technologies Enhanced Learning (CUMTEL)

- Development and applications of digital content for e-learning

- Discipline-based cases for e-learning

- e-Learning Accessibility (EA)

- Emerging tools and technologies for e-learning

- Game-based Learning

- Information Society \& Culture (ISC)

- Innovative Design of Learning Software (IDOLS)

- Joyful Learning \& Society (JL\&S)

- Learning and Knowledge Management

- Mobile and Ubiquitous Learning

- Social computing in e-learning

- Technology Enhanced Language Learning (TELL)

- Technology Enhanced Science Learning (TESL)

- Technology Facilitated Testing and Assessment (TFTA)

- Theoretical foundations for e-learning

- Trends in content/courseware development

APTEL 2010 has successfully attracted over 200 submissions, from 17 countries. The acceptance rate of full papers is less than $20 \%$, showing the high quality of this conference. In this special issue, eleven papers related to e-learning and knowledge management are included. For example, the paper "Robustness of BW aberrance indices against test length" presents a behavior analysis model to detect the aberrant responses; both the papers "The Synergy of Paper-Based and Digital Material for Ubiquitous Foreign Language Learners" and "Online Language Teaching: the Pedagogical Challenges" focus on technology-enhanced language learning; the paper "Student Modeling in Adaptive E-Learning Systems" presents a study of developing a adaptive learning system based on a student model; the papers "A Technical Mode for Sharing and Utilizing Open Educational Resources in Chinese Universities", "Adopting Knowledge Management in an E-Learning System: Insights and views of KM and EL Research Scholars" and "Analysis of Relating the Use of a Learning Management System to Teacher Epistemology and Course Characteristics in Higher Education" refer to the experiences of managing and sharing educational resources.

In addition, some papers focus on investigating the effects of technology enhanced learning. For example, both the paper "The Effects of Integrated Information 
Literacy in Science Curriculum on First-Grade Students' Memory and Comprehension Using the Super3 Model" and "Negotiating content with learners using technology enhanced teaching and learning solutions" show some practical applications and the interesting findings with respect to how information technologies can be applied to improve children's learning.

This special issue also includes some emerging topics of technology-enhanced learning. For example, the paper "Usability of Context-Aware Mobile Educational Game" presents a study of developing a mobile educational game with sensing technology; the paper "Effects of Stereoscopic 3D Contents on the Process of Learning to build a Handmade PC" presents the development of 3D content for a computer course.

From the topics of these papers, it can be seen that this special issue has covered almost all of the well recognized issues of e-learning and knowledge management, including behavior pattern analysis, computer-assisted langrage learning, mobile learning, game-based learning, adaptive learning, computer-assisted testing and educational resource management. Based on the papers coved in this special issue, one can also sense that the impact of ICT on education is shifting from technology-enhanced learning to technology transformed learning. 